\title{
Optimal CDMA Signatures: A Finite-Step Approach
}

\author{
Joel A. Tropp \\ Inst. for Comp. Engr. and Sci. (ICES) \\ The University of Texas at Austin \\ 1 University Station C0200 \\ Austin, TX 78712 \\ jtropp@ices.utexas.edu
}

\author{
Inderjit. S. Dhillon \\ Dept. of Comp. Sci. \\ The University of Texas at Austin \\ 1 University Station C0500 \\ Austin, TX 78712 \\ inderjit@cs.utexas.edu
}

\author{
Robert W. Heath Jr. \\ Dept. of Elect. and Comp. Engr. \\ The University of Texas at Austin \\ 1 University Station C0803 \\ Austin, TX 78712-1084 USA \\ rheatheece.utexas. edu
}

\begin{abstract}
A description of optimal sequences for direct-spread code division multiple access is a byproduct of recent characterizations of the sum capacity. This papers restates the sequence design problem as an inverse singular value problem and shows that it can be solved with finite-step algorithms from matrix analysis. Relevant algorithms are reviewed and a new one-sided construction is proposed that obtains the sequences directly instead of computing the Gram matrix of the optimal signatures.
\end{abstract}

\section{INTRODUCTION}

The problem of sequence sequences to maximize the sum capacity of symbol-synchronous direct-spread code division multiple access system (henceforth S-CDMA) has received significant attention in the information theory community over the last decade, e.g. [1]-[7]. Despite this work, the algorithms proposed to find optimal signatures have not exploited the observation that the signature design problem can be characterized as an inverse singular value problem [8]. Thus researchers have been unable to exploit the wealth of algorithms developed in the matrix theory community during the past two decades [9], [10].

In this paper we present the signature design problem from a new perspective: as the solution to an inverse singular value problem. In short, optimal sequences are intimately connected to matrices that satisfy certain conditions on their column norms and singular spectrum; these constraints are determined by the user power requirements and the noise covariance. This matrix analysis point-of-view allows us to leverage existing algorithms for solving inverse singular value problems to develop numerically stable, finite algorithms for solving the sequence design problem. Unlike iterative algorithms, e.g. [4], [7], [11], convergence is not an issue for finite-step algorithms since they are guaranteed to solve the stated problem.

The specific contributions of this paper are two-fold. First we present a summary of relevant finite-step algorithms from matrix analysis that will be useful for researchers working in sequence design. We focus on the algorithms by BendelMickey [9], Chan-Li [10] and Davies-Higham [12]. Second, we present a new one-sided, finite algorithm that produces the signature sequences directly instead of computing their Gram matrix. This method has very modest time and space requirements in comparison with the other techniques we discuss. Throughout we focus on the case of complex signatures exclusively because it subsumes the real case without any additional difficulty of argument.

\section{BACKGROUND}

\section{A. Synchronous DS-CDMA}

Consider the uplink of an S-CDMA system with $N$ users and a processing gain of $d$. Assume that $N>d$, since the analysis of the other case is straightforward. Define a $d \times N$ matrix whose columns are the signatures: $S \stackrel{\text { def }}{=}\left[\begin{array}{lllll}s_{1} & s_{2} & \ldots & s_{N}\end{array}\right]$. Let $S^{*}$ to denote the (conjugate) transpose of $S$. Note that $\left(S^{*} S\right)_{n n}=1$ for each $n=1, \ldots, N$. Assume that user $n$ has an average power $w_{n}$ and collect them in the diagonal matrix $W \stackrel{\text { def }}{=} \operatorname{diag}\left(w_{1}, w_{2}, \ldots, w_{N}\right)$. It is often more convenient to absorb the power constraints into the signatures, so we also define the weighted signature matrix $X \stackrel{\text { def }}{=} S W^{1 / 2}$. Denote the $n$-th column of $X$ as $\boldsymbol{x}_{n}$. For each $n$, one has the relationship

$$
\left(X^{*} X\right)_{n n}=\left\|\boldsymbol{x}_{n}\right\|_{2}^{2}=w_{n} .
$$

Finally, let $\Sigma$ denote the covariance matrix of the noise.

Viswanath and Anantharam have proven in [5] that, for real signatures, the sum capacity of the S-CDMA channel per degree of freedom is given by the expression (in the complex case, the sum capacity differs by a constant factor)

$$
C_{\text {sum }}=\frac{1}{2 d} \max _{S} \log \operatorname{det}\left(\mathrm{I}_{d}+\Sigma^{-1} S W S^{*}\right)
$$

The basic sequence design problem is to produce a signature matrix $S$ that solves the optimization problem (2). Various specializations have been considered with equal user powers, white noise, unequal user powers, and colored noise. We will show how each of these cases results in an inverse singular value problem.

\section{B. A Sum Capacity Bound}

In [1], Rupf and Massey produced an upper bound on the sum capacity under white noise with variance $\sigma^{2}$ :

$$
C_{\text {sum }} \leq \frac{1}{2} \log \left(1+\frac{\operatorname{Tr} W}{\sigma^{2} d}\right)
$$

where $\operatorname{Tr}(\cdot)$ indicates the trace operator. They also established a necessary and sufficient condition on the signatures for equality to be attained in the bound (3):

$$
X X^{*}=S W S^{*}=\frac{\operatorname{Tr} W}{d} \mathrm{I}_{d}
$$

A matrix $X$ that satisfies (4) is known as a tight frame [13] or a general Welch-Bound-Equality sequence (gWBE) [2]. A 
condition equivalent to (4) is that

$$
X^{*} X=\frac{\operatorname{Tr} W}{d} P
$$

where the matrix $P$ represents an orthogonal projector from $\mathbb{C}^{N}$ onto a subspace of dimension $d$. Recall that an orthogonal projector is an idempotent, Hermitian matrix. That is, $P^{2}=P$ and $P=P^{*}$. An orthogonal projector is also characterized as a Hermitian matrix whose nonzero eigenvalues are identically equal to one. In light of equation (1), the problem of constructing optimal signature sequences in the present setting is closely related to the problem of constructing an orthogonal projector with a specified diagonal.

\section{White Noise, Equal Powers}

Consider the case where the power constraints are equal, viz. $W=w I_{N}$ for some positive number $w$. Then condition (4) for equality to hold in (3) becomes

$$
w^{-1} X X^{*}=S S^{*}=\frac{N}{d} \mathrm{I}_{d} .
$$

A matrix $S$ which satisfies (6) is known as a unit-norm tight frame (UNTF) [13] or a Welch-Bound-Equality sequence (WBE) [1]. In fact, there always exist signature matrices that satisfy condition (6), and so the upper bound on the sum capacity can always be attained when the users' power constraints are equal [1]. The equation (6) can also be interpreted as a restriction on the singular values of the signature matrix. Under the assumptions of white noise and equal power constraints, a matrix $S$ yields optimal signatures if and only if

1) each column of $S$ has unit-norm and

2) the $d$ nonzero singular values of $S$ are identically equal to $\sqrt{N / d}$.

Therefore, this sequence design problem falls into the category of structured inverse singular value problems. Note that condition 1) must hold irrespective of the type of noise.

\section{Majorization}

The bound (3) cannot be met for an arbitrary set of power constraints. The explanation requires some background in majorization [14]. Essentially, majorization defines a partial ordering that allows two vectors of equal norm to be compared. A vector $\boldsymbol{w}$ majorizes $\boldsymbol{\lambda}$ (written $\boldsymbol{w} \succcurlyeq \boldsymbol{\lambda}$ ) if all the partial sums of the entries of $\boldsymbol{w}$ are greater than or equal to the partial sums of $\boldsymbol{\lambda}$. It turns out that majorization defines the precise relationship between the diagonal entries of a Hermitian matrix and its spectrum (see [14], [15] for details).

Theorem 1 (Schur-Horn [15]): The diagonal entries of a Hermitian matrix majorize its eigenvalues. Conversely, if $\boldsymbol{w} \succcurlyeq$ $\lambda$, there exists a Hermitian matrix with diagonal elements listed by $\boldsymbol{w}$ and eigenvalues listed by $\boldsymbol{\lambda}$.

\section{E. White Noise, Unequal Powers}

The Schur-Horn Theorem forbids the construction of an orthogonal projector with arbitrary diagonal entries. For this reason, (5) cannot always hold, and the upper bound (3) cannot always be attained.
The key result of [2] is a complete characterization of the sum capacity of the S-CDMA channel under white noise. Viswanath and Anantharam demonstrate that oversized users-those whose power constraints are too large relative to the others for the majorization condition to hold-must receive their own orthogonal channels to maximize the sum capacity of the system, and they provide a simple method of determining which users are oversized. The other users share the remaining dimensions equitably.

Suppose that there are $m<d$ oversized users, whose signatures form the columns of $S_{0}$. Let the columns of $S_{1}$ list the signatures of the $(N-m)$ remaining users, and let the diagonal matrix $W_{1}$ list their power constraints. The conditions for achieving sum capacity follow.

1) The $m$ oversized users receive orthogonal signatures: $S_{0}^{*} S_{0}=I_{m}$.

2) The remaining $(N-m)$ signatures are also orthogonal to the oversized users' signatures: $S_{0}^{*} S_{1}=0$.

3) The remaining users signatures satisfy

$$
S_{1} W_{1} S_{1}^{*}=\frac{\operatorname{Tr} W_{1}}{d-m} \mathrm{I}_{d-m}
$$

Repeat the foregoing arguments to see that the sequence design problem still amounts to constructing a matrix with given column norms and singular spectrum. It is therefore an inverse singular value problem.

\section{F. Colored Noise, Unequal Powers}

When the noise is colored, the situation is somewhat more complicated. Nevertheless, optimal sequence design still boils down to constructing a matrix with given column norms and singular spectrum. Viswanath and Anantharam show that the following procedure will solve the problem [5].

1) Compute an eigenvalue decomposition of the noise covariance matrix, $\Sigma=Q D Q^{*}$, where $D=\operatorname{diag} \sigma$ for some non-negative vector $\sigma$.

2) Use Algorithm $\mathcal{A}$ of [5] to determine $\boldsymbol{\mu}$, the Schurminimal element of the set of possible eigenvalues of $S W S^{*}+\Sigma$.

3) Form the vector $\boldsymbol{\lambda} \stackrel{\text { def }}{=} \boldsymbol{\mu}-\boldsymbol{\sigma}$.

4) Compute an auxiliary signature matrix $T$ with unit-norm columns so that $T W T^{*}=\operatorname{diag} \boldsymbol{\lambda}$.

5) The optimal signature matrix is $S \stackrel{\text { def }}{=} Q T$.

The computation in step (4) is equivalent to producing a $d \times N$ matrix $X \stackrel{\text { def }}{=} T W^{1 / 2}$. The columns of $X$ must have squared norms listed by the diagonal of $W$. The vector $\lambda$ must list the $d$ nonzero squared singular values of $X$. This is another inverse singular value problem.

\section{Constructing Correlation Matrices}

A positive semi-definite Hermitian matrix with a unit diagonal is known as a correlation matrix [12]. The Gram matrix $A \stackrel{\text { def }}{=} S^{*} S$ of an optimal signature matrix $S$ is always a correlation matrix. Every correlation matrix with the appropriate spectrum can be factored to produce optimal signature matrices [12]. Therefore, we begin with a technique for constructing correlation matrices with a preassigned spectrum. 


\section{A. The Bendel-Mickey Algorithm}

In 1978, Bendel and Mickey presented an algorithm that uses a finite sequence of rotations to convert an arbitrary $N \times N$ Hermitian matrix with trace $N$ into a unit-diagonal matrix that has the same spectrum [9]. We follow the superb exposition of Davies and Higham [12]. Brief discussions appear on page 76 of Horn and Johnson [15] and in Problems 8.4.1 and 8.4.2 of Golub and van Loan [16].

Suppose that $A \in \mathbb{M}_{N}$ is a Hermitian matrix with $\operatorname{Tr} A=$ $N$. (Let $\mathbb{M}_{N}$ denote the set of complex $N \times N$ matrices and $\mathbb{M}_{d, N}$ to denote the set of complex $d \times N$ matrices.) If $A$ does not have a unit diagonal, one can locate two diagonal elements so that $a_{j j}<1<a_{k k}$; otherwise, the trace condition would be violated. It is then possible to construct a real plane rotation $Q$ in the $j k$-plane so that $\left(Q^{*} A Q\right)_{j j}=1$. The transformation $A \mapsto Q^{*} A Q$ preserves the conjugate symmetry and the spectrum of $A$ but reduces the number of non-unit diagonal entries by at least one. Thus, at most $(N-1)$ rotations are required before the resulting matrix has a unit diagonal.

The appropriate form of the rotation is easy to discover, but the following derivation is essential to ensure numerical stability. Recall that a two-dimensional plane rotation is an orthogonal matrix of the form

$$
Q=\left[\begin{array}{cc}
c & s \\
-s & c
\end{array}\right]
$$

where $c^{2}+s^{2}=1$ [16]. The corresponding plane rotation in the $j k$-plane is the $N$-dimensional identity matrix with its $j j, j k, k j$ and $k k$ entries replaced by the entries of the twodimensional rotation. Let $j<k$ be indices so that

$$
a_{j j}<1<a_{k k} \quad \text { or } \quad a_{k k}<1<a_{j j} .
$$

The desired plane rotation yields the matrix equation

$$
\left[\begin{array}{cc}
c & s \\
-s & c
\end{array}\right]^{*}\left[\begin{array}{ll}
a_{j j} & a_{j k} \\
a_{j k}^{*} & a_{k k}
\end{array}\right]\left[\begin{array}{cc}
c & s \\
-s & c
\end{array}\right]=\left[\begin{array}{cc}
1 & \widetilde{a}_{j k} \\
\widetilde{a}_{j k}^{*} & \widetilde{a}_{k k}
\end{array}\right]
$$

where $c^{2}+s^{2}=1$. The equality of the upper-left entries can be stated as

$$
c^{2} a_{j j}-2 s c \operatorname{Re} a_{j k}+s^{2} a_{k k}=1 .
$$

This equation is quadratic in $t=s / c$ :

$$
\left(a_{k k}-1\right) t^{2}-2 t \operatorname{Re} a_{j k}+\left(a_{j j}-1\right)=0
$$

whence

$$
t=\frac{\operatorname{Re} a_{j k} \pm \sqrt{\left(\operatorname{Re} a_{j k}\right)^{2}-\left(a_{j j}-1\right)\left(a_{k k}-1\right)}}{a_{k k}-1} .
$$

Notice that the choice of $j$ and $k$ guarantees a positive discriminant. As is standard in numerical analysis, the \pm sign in (7) must be taken to avoid cancelations. If necessary, one can extract the other root using the fact that the product of the roots equals $\left(a_{j j}-1\right) /\left(a_{k k}-1\right)$. Finally,

$$
c=\frac{1}{\sqrt{1+t^{2}}} \quad \text { and } \quad s=c t .
$$

Floating-point arithmetic is inexact, so the rotation may not yield $a_{j j}=1$. A better implementation sets $a_{j j}=1$ explicitly. Davies and Higham prove that the Bendel-Mickey algorithm is backward stable, so long as it is implemented the way we have described [12]. We restate the algorithm.

Algorithm 1 (Bendel-Mickey): Given Hermitian $A \in \mathbb{M}_{N}$ with $\operatorname{Tr} A=N$, this algorithm yields a correlation matrix whose eigenvalues are identical with those of $A$.

1) While some diagonal entry $a_{j j} \neq 1$, repeat Steps $2-4$.

2) Find an index $k$ (without loss of generality $j<k$ ) for which $a_{j j}<1<a_{k k}$ or $a_{k k}<1<a_{j j}$.

3) Determine a plane rotation $Q$ in the $j k$-plane using equations (7) and (8).

4) Replace $A$ by $Q^{*} A Q$. Set $a_{j j}=1$.

Since the loop executes no more than $(N-1)$ times, the total cost of the algorithm is no more $12 N^{2}$ real floating-point operations, to highest order, if conjugate symmetry is exploited. The plane rotations never need to be generated explicitly, and all the intermediate matrices are Hermitian. Therefore, the algorithm must store only $N(N+1) / 2$ complex floatingpoint numbers. MATLAB 6 contains a version of Algorithm 1 that starts with a random matrix of specified spectrum. The command is gallery ('randcorr', ...).

It should be clear that a similar algorithm can be applied to any Hermitian matrix $A$ to produce another Hermitian matrix with the same spectrum but whose diagonal entries are identically equal to $\operatorname{Tr} A / N$.

The columns of $S^{*}$ must form an orthogonal basis for the column space of $A \stackrel{\text { def }}{=} S^{*} S$ according to (6). Therefore, one can use a rank-revealing $\mathrm{QR}$ factorization to extract a signature sequence $S$ from the output $A$ of Algorithm 1 [16].

\section{B. The Davies-Higham Algorithm}

The methods of the last section can be modified to compute the signature sequence directly without recourse to an additional $\mathrm{QR}$ factorization. Any correlation matrix $A \in \mathbb{M}_{N}$ can be expressed as the product $S^{*} S$ where $S \in \mathbb{M}_{r, N}$ has columns of unit norm and dimension $r \geq \operatorname{rank} A$. With this factorization, the two-sided transformation $A \mapsto Q^{*} A Q$ is equivalent to a one-sided transformation $S \mapsto S Q$. In consequence, the machinery of the Bendel-Mickey algorithm requires little adjustment to produce these factors. We refer to the one-sided version as the Davies-Higham algorithm in view of [12]. We have observed that it can also be used to find the factors of an $N$-dimensional correlation matrix with rank $r<N$, in which case $S$ may take dimensions $d \times N$ for any $d \geq r$.

Algorithm 2 (Davies-Higham): Given $S \in \mathbb{M}_{d, N}$ for which $\operatorname{Tr} S^{*} S=N$, this procedure yields a $d \times N$ matrix with the same singular values as $S$ but with unit-norm columns.

1) Calculate and store the column norms of $S$.

2) While some column has norm $\left\|s_{j}\right\|_{2}^{2} \neq 1$, repeat Steps 3-7.

3) Find indices $j<k$ for which

$$
\left\|\boldsymbol{s}_{j}\right\|_{2}^{2}<1<\left\|\boldsymbol{s}_{k}\right\|_{2}^{2} \quad \text { or } \quad\left\|\boldsymbol{s}_{k}\right\|_{2}^{2}<1<\left\|\boldsymbol{s}_{j}\right\|_{2}^{2} .
$$


4) Form the quantities

$$
a_{j j}=\left\|s_{j}\right\|_{2}^{2}, \quad a_{j k}=\left\langle s_{k}, s_{j}\right\rangle \quad \text { and } \quad a_{k k}=\left\|s_{k}\right\|_{2}^{2} .
$$

5) Determine a rotation $Q$ in the $j k$-plane using equations (7) and (8).

6) Replace $S$ by $S Q$.

7) Update the two column norms that have changed.

Step (1) requires $4 d N$ real floating-point operations, and the remaining steps require $12 d N$ real floating-point operations to highest order. The algorithm requires the storage of $d N$ complex floating-point numbers and $N$ real numbers for the current column norms. Davies and Higham remark that the algorithm is numerically stable [12].

\section{CHAN-Li AND ITS VARIANTS}

Now we discuss a simple technique for constructing a limited selection of Hermitian matrices with prescribed diagonal and spectrum. This is the core problem for the unequal power case with white or colored noise because every optimal weighted signature sequence has a Gram matrix $A \stackrel{\text { def }}{=} X^{*} X$ with fixed diagonal and spectrum (and conversely). The algorithm begins with a diagonal matrix of eigenvalues and applies a sequence of rotations to impose the power constraints. A similar technique can be used to build optimal weighted signature sequences $X$ directly.

\section{A. The Chan-Li Algorithm}

Chan and $\mathrm{Li}$ present a beautiful, constructive proof of converse part of the Schur-Horn Theorem [10]. Suppose that $\boldsymbol{w}$ and $\boldsymbol{\lambda}$ are $N$-dimensional, real vectors for which $\boldsymbol{w} \succcurlyeq \boldsymbol{\lambda}$. Using induction on the dimension, we show how to construct a Hermitian matrix with diagonal $\boldsymbol{w}$ and spectrum $\boldsymbol{\lambda}$. In the sequel, assume without loss of generality that the entries of $\boldsymbol{w}$ and $\boldsymbol{\lambda}$ have been sorted in ascending order. Therefore, $w_{(k)}=w_{k}$ and $\lambda_{(k)}=\lambda_{k}$ for each $k$.

Suppose that $N=2$. The majorization relation implies $\lambda_{1} \leq$ $w_{1} \leq w_{2} \leq \lambda_{2}$. Let $A \stackrel{\text { def }}{=} \operatorname{diag} \boldsymbol{\lambda}$. We can explicitly construct a plane rotation $Q$ so that the diagonal of $Q^{*} A Q$ equals $\boldsymbol{w}$ :

$$
Q \stackrel{\text { def }}{=} \frac{1}{\sqrt{\lambda_{2}-\lambda_{1}}}\left[\begin{array}{cc}
\sqrt{\lambda_{2}-w_{1}} & \sqrt{w_{1}-\lambda_{1}} \\
-\sqrt{w_{1}-\lambda_{1}} & \sqrt{\lambda_{2}-w_{1}}
\end{array}\right] .
$$

Since $Q$ is orthogonal, $Q^{*} A Q$ retains spectrum $\lambda$ but gains diagonal entries $\boldsymbol{w}$.

Suppose that, whenever $\boldsymbol{w} \succcurlyeq \boldsymbol{\lambda}$ for vectors of length $N-$ 1 , we can construct an orthogonal transformation $Q$ so that $Q^{*}(\operatorname{diag} \boldsymbol{\lambda}) Q$ has diagonal entries $\boldsymbol{w}$.

Consider $N$-dimensional vectors for which $\boldsymbol{w} \succcurlyeq \boldsymbol{\lambda}$. Let $A \stackrel{\text { def }}{=}$ $\operatorname{diag} \boldsymbol{\lambda}$. The majorization condition implies that $\lambda_{1} \leq w_{1} \leq$ $w_{N} \leq \lambda_{N}$, so it is always possible to select a least integer $j>1$ so that $\lambda_{j-1} \leq w_{1} \leq \lambda_{j}$. Let $P_{1}$ be a permutation matrix for which

$$
P_{1}^{*} A P_{1}=\operatorname{diag}\left(\lambda_{1}, \lambda_{j}, \lambda_{2}, \ldots, \lambda_{j-1}, \lambda_{j+1}, \ldots, \lambda_{N}\right) .
$$

Observe that $\lambda_{1} \leq w_{1} \leq \lambda_{j}$ and $\lambda_{1} \leq \lambda_{1}+\lambda_{j}-w_{1} \leq$ $\lambda_{j}$. Thus we may use equation (9), replacing $\lambda_{2}$ with $\lambda_{j}$, to construct a plane rotation $Q_{2}$ that sets the first entry of $Q_{2}^{*}\left(\operatorname{diag}\left(\lambda_{1}, \lambda_{j}\right)\right) Q_{2}$ to $w_{1}$. If we define the rotation

$$
P_{2} \stackrel{\text { def }}{=}\left[\begin{array}{cc}
Q_{2} & 0^{*} \\
0 & \mathrm{I}_{N-2}
\end{array}\right]
$$

then

$$
P_{2}^{*} P_{1}^{*} A P_{1} P_{2}=\left[\begin{array}{cc}
w_{1} & \boldsymbol{v}^{*} \\
\boldsymbol{v} & A_{N-1}
\end{array}\right]
$$

where $\boldsymbol{v}$ is an appropriate vector and $A_{N-1}=$ $\operatorname{diag}\left(\lambda_{1}+\lambda_{j}-w_{1}, \lambda_{2}, \ldots, \lambda_{j-1}, \lambda_{j+1}, \ldots, \lambda_{N}\right)$.

To apply the induction hypothesis, it remains to check that the vector $\left(w_{2}, w_{3}, \ldots, w_{N}\right)$ majorizes the diagonal of $A_{N-1}$. We accomplish this in three steps. First, recall that $\lambda_{k} \leq w_{1}$ for $k=2, \ldots, j-1$. Therefore,

$$
\sum_{k=2}^{m} w_{k} \geq(m-1) w_{1} \geq \sum_{k=2}^{m} \lambda_{k}
$$

for each $m=2, \ldots, j-1$. The sum on the right-hand side obviously exceeds the sum of the smallest $(m-1)$ entries of $\operatorname{diag} A_{N-1}$, so the first $(j-2)$ majorization inequalities are in force. Second, use the fact that $\boldsymbol{w} \succcurlyeq \boldsymbol{\lambda}$ to calculate that

$$
\begin{aligned}
\sum_{k=2}^{m} w_{k} & =\sum_{k=1}^{m} w_{k}-w_{1} \geq \sum_{k=1}^{m} \lambda_{k}-w_{1} \\
& =\left(\lambda_{1}+\lambda_{j}-w_{1}\right)+\sum_{k=2}^{j-1} \lambda_{k}+\sum_{k=j+1}^{m} \lambda_{k}
\end{aligned}
$$

for $m=j, \ldots, N$. Once again, observe that the sum on the right-hand side exceeds the sum of the smallest $(m-1)$ entries of $\operatorname{diag} A_{N-1}$, so the remaining majorization inequalities are in force. Finally, rearranging the relation $\sum_{k=1}^{N} w_{k}=$ $\sum_{k=1}^{N} \lambda_{k}$ yields $\sum_{k=2}^{N} w_{k}=\operatorname{Tr} A_{N-1}$.

In consequence, the induction furnishes a rotation $Q_{N-1}$ which sets the diagonal entries of $A_{N-1}$ equal to the numbers $\left(w_{2}, \ldots, w_{N}\right)$. Define

$$
P_{3} \stackrel{\text { def }}{=}\left[\begin{array}{cc}
1 & \mathbf{0}^{*} \\
\mathbf{0} & Q_{N-1}
\end{array}\right] .
$$

Conjugating $A$ by the orthogonal matrix $P=P_{1} P_{2} P_{3}$ transforms the diagonal entries of $A$ to $\boldsymbol{w}$ while retaining the spectrum $\lambda$. The proof yields the following algorithm.

Algorithm 3 (Chan-Li): Let $\boldsymbol{w}$ and $\boldsymbol{\lambda}$ be vectors with ascending entries and such that $\boldsymbol{w} \succcurlyeq \boldsymbol{\lambda}$. The following procedure computes a real, symmetric matrix with diagonal entries $\boldsymbol{w}$ and eigenvalues $\lambda$.

1) Initialize $A=\operatorname{diag} \boldsymbol{\lambda}$, and put $n=1$.

2) Find the least $j>n$ so that $a_{j-1, j-1} \leq w_{n} \leq a_{j j}$.

3) Use a symmetric permutation to set $a_{n+1, n+1}$ equal to $a_{j j}$ while shifting diagonal entries $n+1, \ldots, j-1$ one place down the diagonal.

4) Define a rotation $Q$ in the $(n, n+1)$-plane with

$$
c=\sqrt{\frac{a_{n+1, n+1}-w_{n}}{a_{n+1, n+1}-a_{n n}}}, \quad s=\sqrt{\frac{w_{n}-a_{n n}}{a_{n+1, n+1}-a_{n n}}} .
$$

5) Replace $A$ by $Q^{*} A Q$. 
6) Use a symmetric permutation to re-sort the diagonal entries of $A$ in ascending order.

7) Increment $n$, and repeat Steps 2-7 while $n<N$.

This algorithm requires about $6 N^{2}$ real floating-point operations. It requires the storage of about $N(N+1) / 2$ real floating-point numbers, including the vector $\boldsymbol{w}$. It is conceptually simpler to perform the permutations described in the algorithm, but it can be implemented without them.

We have observed that the algorithm given by Viswanath and Anantharam [2] for constructing gWBEs is similar to the Chan-Li algorithm. Nevertheless, we feel that the simplicity of Chan and Li's presentation merits repetition.

\section{B. One-Sided Chan-Li}

The Chan-Li algorithm only produces a Gram matrix, which must be factored to obtain the weighted signature matrix. Referring back to the ideas of Davies and Higham, we propose a one-sided version of the Chan-Li algorithm. The benefits are several. It requires less storage and less computation than the Chan-Li algorithm. At the same time, it constructs the factors explicitly. This algorithm has not been published before.

Algorithm 4 (One-Sided Chan-Li): Suppose that $\boldsymbol{w}$ and $\boldsymbol{\lambda}$ are non-negative vectors of length $N$ with ascending entries. Assume, moreover, that the first $(N-d)$ components of $\boldsymbol{\lambda}$ are zero and that $\boldsymbol{w} \succcurlyeq \boldsymbol{\lambda}$. The following algorithm produces a $d \times N$ matrix $X$ whose column norms are listed by $\boldsymbol{w}$ and whose squared singular values are listed by $\lambda$.

1) Initialize $n=1$, and set

$$
X=\left[\begin{array}{l|lll}
0 & \sqrt{\lambda_{N-d+1}} & & \\
& & \ddots & \\
& & & \sqrt{\lambda_{N}}
\end{array}\right] .
$$

2) Find the least $j>n$ so that $\left\|\boldsymbol{x}_{j-1}\right\|_{2}^{2} \leq w_{n} \leq\left\|\boldsymbol{x}_{j}\right\|_{2}^{2}$.

3) Move the $j$-th column of $X$ to the $(n+1)$-st column, shifting the displaced columns to the right.

4) Define a rotation $Q$ in the $(n, n+1)$-plane with

$$
c=\sqrt{\frac{\left\|\boldsymbol{x}_{n+1}\right\|_{2}^{2}-w_{n}}{\left\|\boldsymbol{x}_{n+1}\right\|_{2}^{2}-\left\|\boldsymbol{x}_{n}\right\|_{2}^{2}}}, \quad s=\sqrt{\frac{w_{n}-\left\|\boldsymbol{x}_{n}\right\|_{2}^{2}}{\left\|\boldsymbol{x}_{n+1}\right\|_{2}^{2}-\left\|\boldsymbol{x}_{n}\right\|_{2}^{2}}} .
$$

5) Replace $X$ by $X Q$.

6) Sort columns $(n+1), \ldots, N$ in order of increasing norm.

7) Increment $n$, and repeat Steps $2-7$ while $n<N$.

The algorithm requires $3 d N$ real floating-point operations and storage of $N(d+2)$ real floating-point numbersm including the desired column norms and the current column norms. The procedure can be implemented without permutations.

\section{DisCUSION}

We have discussed a group of four algorithms that can be used to produce sum-capacity-optimal S-CDMA sequences in a wide variety of circumstances. The first algorithm, BendelMickey, constructs a Hermitian matrix with a constant diagonal and a prescribed spectrum. This matrix can be factored to yield an optimal signature sequence for the case of equal user powers, i.e. a unit-norm tight frame. Alternately, the DaviesHigham algorithm can be used to produce the factors directly. The third algorithm, Chan-Li, constructs a Hermitian matrix with an arbitrary diagonal and prescribed spectrum, subject to the majorization condition. The resulting matrix can be factored to obtain an optimal signature sequence for the case of unequal received powers, i.e. a tight frame. We have also introduced a new variant, the one-sided Chan-Li algorithm, that can calculate the factors directly.

The Bendel-Mickey and Davies-Higham algorithms can potentially calculate every correlation matrix and its factors. If they are initialized with random matrices, one may interpret the output as a random correlation matrix. The factors can be interpreted as random unit-norm signature sequences.

On the other hand, the output of the Chan-Li and one-sided Chan-Li algorithms is not encyclopedic. They can construct only a few matrices for each pair $(\boldsymbol{w}, \boldsymbol{\lambda})$. These matrices are also likely to have many zero entries, which is undesirable for some applications. In addition, these algorithms only build real matrices, whereas complex matrices are often of more interest.

\section{REFERENCES}

[1] M. Rupf and J. L. Massey, "Optimum sequence multisets for synchronous code-division multiple-access channels," IEEE Trans. Inform. Theory, vol. 40, no. 4, pp. 1261-1266, July 1994.

[2] P. Viswanath and V. Anantharam, "Optimal sequences and sum capacity of synchronous CDMA systems," IEEE Trans. Inform. Th., vol. 45, no. 6 , pp. 1984-1991, Sept. 1999.

[3] S. Ulukus and R. D. Yates, "Iterative construction of optimum signature sequence sets in synchronous CDMA systems," IEEE Trans. Inform. Theory, vol. 47, no. 5, pp. 1989-1998, 2001.

[4] C. Rose, "CDMA codeword optimization: Interference avoidance and convergence via class warfare," IEEE Trans. IT, vol. 47, no. 6, pp. 23682382, 2001.

[5] P. Viswanath and A. Anantharam, "Optimal sequences for CDMA under colored noise: A Schur-saddle function property," IEEE Trans. IT, vol. 48, no. 6, pp. 1295-1318, June 2002.

[6] P. Anigstein and V. Anantharam, "Ensuring convergence of the MMSE iteration for interference avoidance to the global optimum," IEEE Trans. IT, vol. 49, no. 4, pp. 873-885, April 2003.

[7] J. A. Tropp, R. W. Heath Jr., and T. Strohmer, "Optimal CDMA signature sequences, inverse eigenvalue problems and alternating projection," in Proceedings of the 2003 IEEE International Symposium on Information Theory, Yokohama, July 2003, p. 407.

[8] M. T. Chu and G. H. Golub, "Structured inverse eigenvalue problems," Acta Numerica, 2001.

[9] R. B. Bendel and M. R. Mickey, "Population correlation matrices for sampling experiments," Commun. Statist. Simul. Comp., vol. B7, no. 2, pp. 163-182, 1978.

[10] N. N. Chan and K.-H. Li, "Diagonal elements and eigenvalues of a real symmetric matrix," J. Math. Anal. Appl., vol. 91, pp. 562-566, 1983.

[11] S. Ulukus and R. D. Yates, "Iterative construction of optimum signature sequence sets in synchronous CDMA systems," IEEE Trans. IT, vol. 47, no. 5, pp. 1989-1998, 2001.

[12] P. I. Davies and N. J. Higham, "Numerically stable generation of correlation matrices and their factors," BIT, vol. 40, no. 4, pp. 640-651, 2000.

[13] P. Casazza and J. Kovačević, "Equal-norm tight frames with erasures," Adv. Comp. Math., 2002.

[14] A. W. Marshall and I. Olkin, Inequalities: Theory of Majorization and its Applications. Academic Press, 1979.

[15] R. A. Horn and C. R. Johnson, Matrix Analysis. Cambridge University Press, 1985

[16] G. H. Golub and C. F. Van Loan, Matrix Computations, 3rd ed. Johns Hopkins University Press, 1996. 